\title{
"Why and How it Works": The Development of Hypervalent lodine Reagents as an Illustration for the Collaboration of Chemical Synthesis with Modeling and Simulation
}

\author{
Hans Peter Lüthi and Antonio Togni
}

\begin{abstract}
Much of the focus of quantum chemical modeling and simulation is on understanding chemical phenomena and in assisting experiment to further improve and develop the respective chemistry. Given the computational tools available today, rather than assisting the development of new chemicals, modeling and simulation are in the process of making the step towards guiding experiment towards novel targets. In this article we show how an intense collaboration between chemical synthesis and computation leads to a better understanding of hypervalent iodine reagents and how modeling and simulation may direct the search of novel reagents.
\end{abstract}

Keywords: Computational quantum chemistry · Hypervalent iodine compounds .

Quantitative-structure-property relationships - Rational molecular design · Reaction mechanism · Reactivity

\section{Introduction}

For the past few decades, much of the focus of quantum chemical modeling and simulation was on understanding why a given compound shows the properties that make it an item of interest in catalysis, synthesis, or other areas of chemistry. This process usually starts with experiment bringing "something that works" to the attention of modeling and simulation to learn more about "how it works". Knowing how it works is expected to help improve the compound's properties by means of chemical modification, to find more optimal reaction conditions, or to even find new applications.

The reverse situation, namely modeling presenting novel compounds "that might work", is still in its infancy. Among the main obstacles are the processing (archival, analysis, and exchange) of large amounts of data, the shortage of descriptors, as well as approaches allowing to by-

\footnotetext{
${ }^{*}$ Correspondence: PD Dr. H. P. Lüthi
}

ETH Zürich

Department of Chemistry and Applied Bioscience Vladimir-Prelog-Weg 2

$\mathrm{CH}-8093$ Zürich

Tel.: +4144632 2105

E-mail: luethi@ethz.ch pass the in silico screening of compounds in combinatorial space. Once these issues have been addressed, a new era in modeling and simulation is likely to begin.

In this article, we wish to illustrate how the collaboration between quantum chemical modeling and experiment lead to more insight in how a very powerful reagent works. The reagent, a hypervalent iodine compound (structure $\mathbf{1}$ in Fig. 1), was developed in the laboratory of one of the authors. ${ }^{[1]}$ It is used for the trifluoromethylation of a wide array of $C, N, P, O$, or $S$ centered nucleophiles.[2,3] Soon after the development of the reagent, a fruitful collaboration with modeling and simulation began.<smiles>CC1(C)OI(C(F)(F)F)c2ccccc21</smiles>

1<smiles>[R][IH][R]</smiles><smiles>C1C[I-]P1</smiles><smiles>[2H][I+][2H]</smiles>

2<smiles>[R][I-]([R])[3H]</smiles>

3<smiles>CC(=O)O[Te]1(O)(OC(C)=O)OC(=O)c2ccccc21</smiles>

The work presented is the result of four MSc theses ${ }^{[4-7]}$ and two ongoing doctoral research projects supervised jointly by the authors. The vision of this joint experimental and computational research is not only to better understand the reactivity of iodanes in general, and to improve their performance, but to also open the doors for new chemistries by finding "something new that works".

The compounds shown in Fig. 1 all show a very rich and diverse reactivity: Compound $\mathbf{1}$ is the trifluoromethylation reagent introduced above. Diaryliodonium salts 2 , when transformed with a nucleophile $\mathrm{X}^{-}$to yield the corresponding reactive iodane intermediates $\mathbf{3}$ and $\mathbf{4}$, are versatile
Fig. 1. The array of hypervalent iodane reagents $(1,2,5,6)$ and their reactive intermediates $(\mathbf{3}, \mathbf{4})$ discussed in this article. 
reagents for a number of arylation reactions leading to products RX and R'X. ${ }^{[8]}$ Reagent $\mathbf{5}$ is a bridged aryl ligand derivative of 2 leading, amongst others, to products RR'X and R'RX. ${ }^{[9]}$ Compound 6, a $\lambda^{5}$-iodane, is widely used as an oxidant for the conversion of primary and secondary alcohols to aldehydes and ketones (DessMartin reagent; ${ }^{[10]}$ ).

The commonly used picture for the bonding in $\lambda^{3}$-iodanes such as $\mathbf{1}, \mathbf{3}$ or 4 sees the iodine center involved in a ' 3 -center 4-electron' (3c-4e) and a perpendicular '2-center 2-electron' (2c-2e) bond (Fig. $2)$. The two most electron-withdrawing ligands are typically involved in the $3 \mathrm{c}-4 \mathrm{e}$ bond. The barrier for isomerization, however, is usually low.

For the illustration of the results of this joint research we will focus on three topics:

Controlling selectivity: the transformation of $\mathbf{2}$ with nucleophiles raises the question which aryl group will be functionalized (or which group the nucleophile will be arylated with), i.e. how to exert influence on the product ratios $\mathrm{RX} / \mathrm{R}^{\prime} \mathrm{X}$ for different combinations of $\mathrm{R}, \mathrm{R}^{\prime}$ and $\mathrm{X}$.

Controlling chemoselectivity: departing from iodonium salts with bridged aryl ligands such as $\mathbf{5}$, the situation is more complex. The functionalization of the aryl ligands leading to RR'X or possibly R'RX is now also in competition with the arene coupling, a side reaction yielding doubly bridged RR'. There are also radical reactions possible. Thus, the question of how to control the chemoselectivity of this reagent arises.

Reactant improvement and new chemistry: Nucleophiles transformed with reagent 1 will be trifluoromethylated. Mechanistically, this is expected to happen via reductive elimination of the product from the corresponding iodane intermediate. Understanding the reaction mechanisms and exploring the reactivity of chemical modifications of this and other reagents may open the door to guiding experiment to find "something new that works better" or "something that allows new chemistry" within a much shorter time frame.

\section{Results}

\section{Controlling Selectivity}

For the functionalization reaction starting from diaryliodonium 2 , we were able to correlate the ratio between products RX and R'X with the polarity of the 3c$4 \mathrm{e}$ bonds observed in the reactive intermediates 3 and 4: if $\mathrm{R}$ forms the more polar $3 \mathrm{c}-4 \mathrm{e}$ bond, it will act as 'directing group', thus facilitating the functionalization of $\mathrm{R}^{\prime}$ to $\mathrm{R}^{\prime} \mathrm{X}{ }^{[8]}$

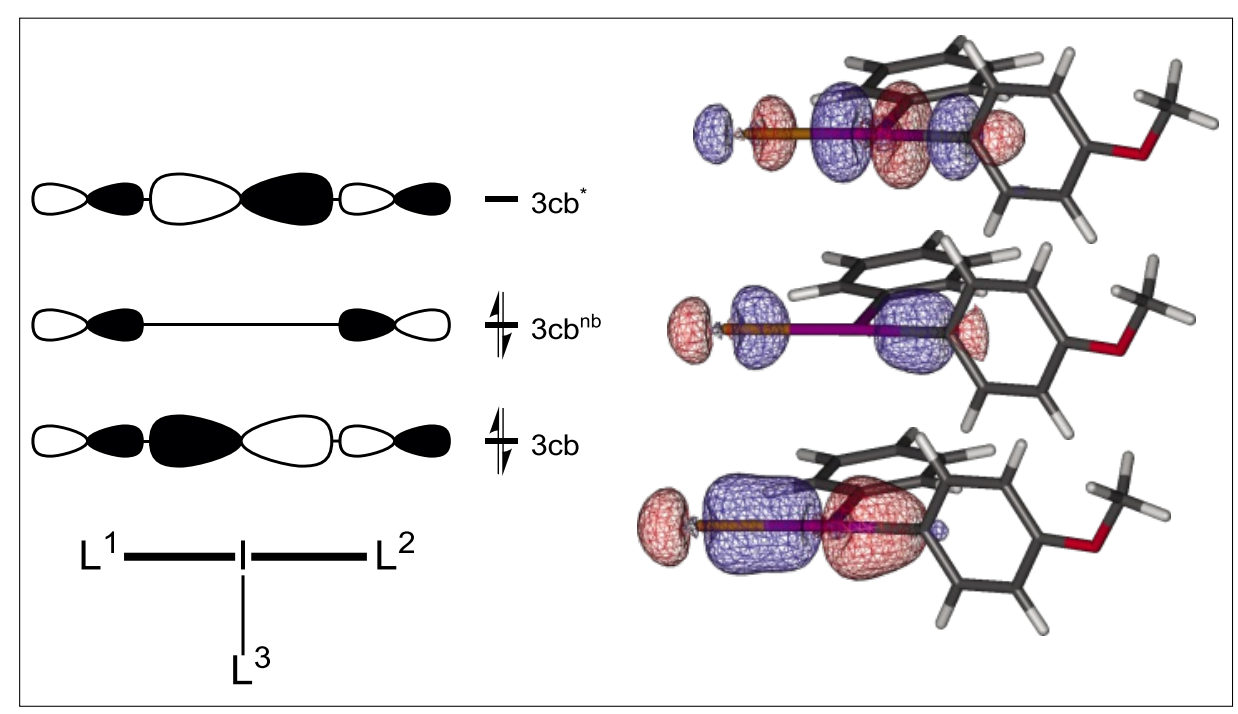

Fig. 2. The $3 c-4 e$ bond in $\lambda^{3}$ ioadanes (schematic representation and corresponding localized orbitals for the bromo $\left(L^{1}\right)$-anisyl $\left(L^{2}\right)$-phenyl $\left(L^{3}\right)$ compound).
Since the reactive intermediates 3 and 4 isomerize rapidly, the reaction mechanism can be expressed in terms of a Curtinnation (RE) leading to the final products as the rate-determining step. The example presented in Fig. 3 shows the profile for 4-methoxyphenyl (or anisyl) and phenyl as aryl ligands $\mathrm{R}$ and $\mathrm{R}^{\prime}$, with $\mathrm{X}^{-}$as a bromide nucleophile. The computed barrier for the RE leading to the functionalization of the phenyl ligand is lower by $2.05 \mathrm{kcal} / \mathrm{mol}$. The observed ratio between $\mathrm{R}^{\prime} \mathrm{X}$ and $\mathrm{RX}$ is $9: 1$. The analysis shows that the $3 c-4 c$ bond formed with the anisyl group (labeled 'anti' in Fig. 3) is more polar than the corresponding bond formed with the phenyl ligand (labeled 'syn'). Expressed in terms of the charges of the ipso carbon atoms of the two ligands ( -0.35 vs. -0.31 electron), the difference amounts to -0.04 electron. This difference in polarity was shown to correlate with the difference in the energies Hammett profile with the reductive elimi- of the respective reaction barriers $\left(\Delta \Delta G^{\#}\right)$ : whereas in the anisyl/phenyl example $\Delta \Delta \mathrm{G}^{\#}$ amounts to $+2.05 \mathrm{kcal} / \mathrm{mol}$, for the 4-trifluoromethyl-phenyl/phenyl pair of ligands we observe a positive charge difference of $\Delta \Delta \mathrm{G} \#$ of $-2.59 \mathrm{kcal} / \mathrm{mol}$, thus favoring the functionalization of the 4-trifluorophenyl group.

This correlation was confirmed and explored further by Olofsson and coworkers. ${ }^{[9]}$ It was shown that the charge of the ipso carbon atom indirectly reflects the inductive effect of the ligand. Plotting the Hammett $\sigma$-values against $\Delta \Delta \mathrm{G}^{\#}$ leads to a robust correlation between the two values (Fig. 4 in ref. [9]). Also, DiMagno had shown that if the directing ligand is decorated with bulky side group, it will eventually lose its capability of electronically directing the reaction and be functionalized even if the second ligand forms a less polar bond.[11] ference $(+0.03$ electron) along with a dif-

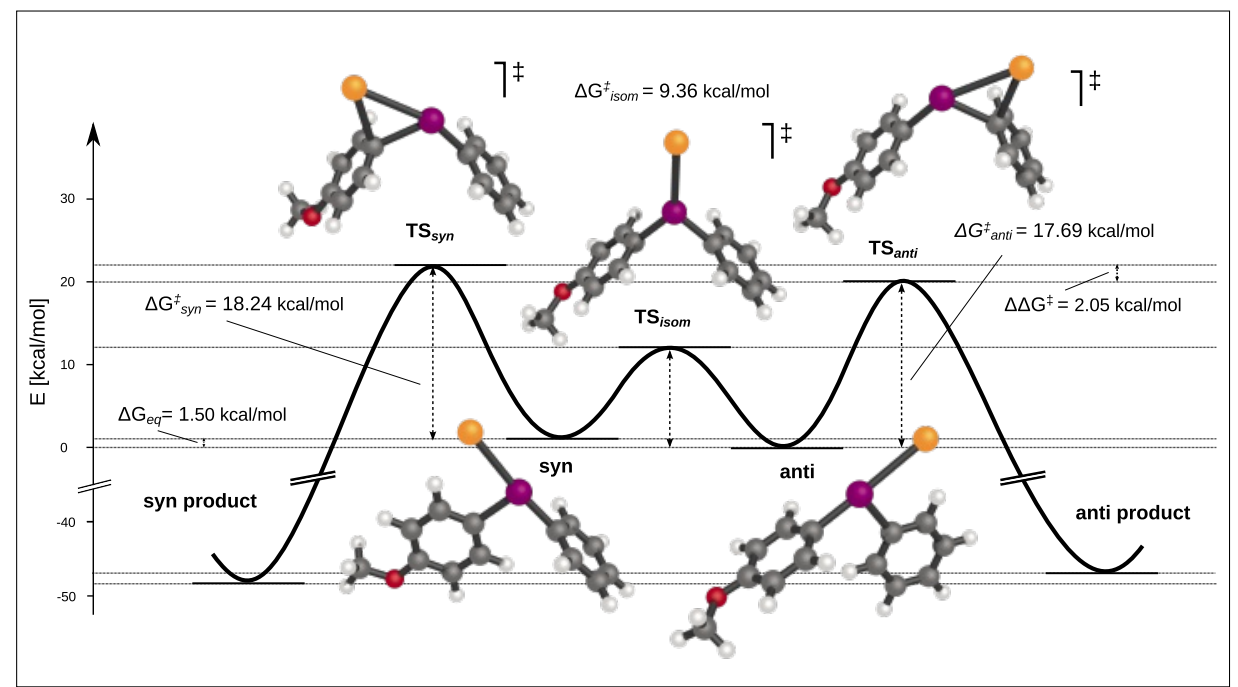

Fig. 3. Reaction profile of bromo-4-methoxyaryl-phenyl-iodane giving the relative barrier heights $\Delta \mathrm{G}^{\ddagger}$ and their absolute differences $\Delta \Delta \mathrm{G}^{\ddagger}$ expressed in terms of Gibbs free energies. Also included are the structures of the stationary points. 


\section{Controlling the Chemoselectivity}

Mechanistic studies on the iodonium reagent with bridged aryl ligands show that the $\lambda^{3}$-iodane formed upon transformation of 5 with a nucleophile takes the role of a 'gateway' as all reaction pathways depart from this intermediate (see structure on gold background in the center of Fig. 4 representing the case of bridge size $n=0$ and RR' = diphenyl). Whereas the isomerization of the iodane intermediate is a low barrier process, vastly unaffected by the size of the bridge, the strain caused by the bridge forces an out-of-plane mechanism for the functionalization reaction: in order to establish a bond to the ipso carbon center, the nucleophile has to be bent out of the molecular plane (see Fig. 4). This outof-plane distortion of the transition state geometry comes with an 'energy penalty' which steadily decreases to finally disappear for unstrained systems. For strained systems, the energy penalty is large enough to make the other reaction channels competitive. ${ }^{[12]}$

The arene coupling reaction (see Fig. 4) also responds to strain, but in a different way: the activation energy shows a minimum for a bridge of size $n=2$, due to a very favorable boat-shape transition state geometry of the heterocycle involving the iodine atom. This favorable transition state geometry is not accessible for the other compounds in the series. At this point $(n=2)$, the arene coupling reaction is the dominant one, showing a computed barrier of $19.6 \mathrm{kcal} / \mathrm{mol}\left(\mathrm{X}=\mathrm{Br}^{-}\right)$; the barrier to functionalization is $21.2 \mathrm{kcal} / \mathrm{mol}$. For larger size bridges, the activation energy for the barrier to arene coupling will increase again, whereas the barrier to functionalization will converge towards 17.3 $\mathrm{kcal} / \mathrm{mol}$.[12]

Radical mechanisms (see Fig. 4) are observed for very strained systems only. Whereas an experimental study suggested a single electron transfer mechanism, ${ }^{[13]}$ we presented homolytic cleavage of the $3 \mathrm{c}$ $4 \mathrm{e}$ bond as plausible alternative. ${ }^{[12]}$ Excited state calculations showed that the cleavage of the $3 \mathrm{c}-4 \mathrm{e}$ bond can be induced by thermal population of the $3 \mathrm{c}-4 \mathrm{e}$ antibonding orbitals. However, the activation barrier is too high to be competitive also for more relaxed systems.

One of the most remarkable features, though, is the affinity of the hypervalent region towards planarity for all reactions. Distortion from planarity was shown to cause an unfavorable interaction between the highest occupied molecular orbital (HOMO) and the iodine lone $5 p$ pair orbitals, resulting in an energy penalty of 10 $\mathrm{kcal} / \mathrm{mol}$ and more. With the loss of planarity there is a reduction of symmetry such that the two orbitals fall into the same irreducible representation, i.e. a pseudo Jahn-

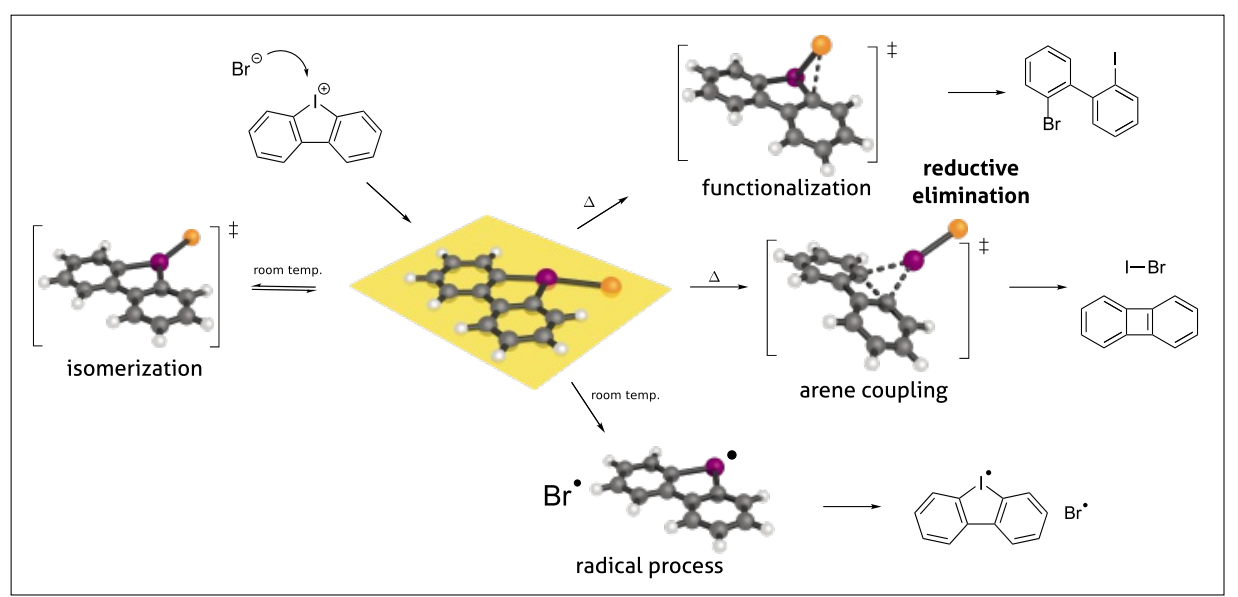

Fig. 4. The reactive intermediate (center) and its role as 'gateway' for three competing reaction channels.
Teller effect situation emerging. There is a one-to-one correlation between the out of plane angle and the energy penalty. ${ }^{[12]}$

\section{Reactant Improvement and Towards New Chemistries}

The focus of the early experimental work on the trifluoromethylation reagent 1 was on the exploration of the chemistry it allows for, i.e. its transformation with an array of nucleophiles ranging from simple alcohols to small peptides. At the same time, some consideration also went into the optimization of the reaction conditions and on the issue that once heated above 70 ${ }^{\circ} \mathrm{C}$, the reagent would decompose rapidly.

The study of the decomposition of the reagent and the search for its stabilization through chemical modification was the topic of our first joint research project. ${ }^{[4]}$ The $a b$ initio molecular dynamics (AIMD) calculations performed by R. Helbling, somewhat surprisingly, showed that the decomposition is not a mono-, but most also showed that protonation of the oxygen atom substantially reduces the stability of the $3 \mathrm{c}-4 \mathrm{e}$ bond. This would not necessarily render an explanation for its decomposition, but possibly explain the enhanced reactivity of the reagent in acidic medium observed in the context of the trifluoromethylation of sulfonic acids. ${ }^{[2,14]}$

Later studies ${ }^{[4]}$ showed that the protonation of the oxygen atom in $\mathbf{1}$ results in a change of the character of the lowest unoccupied orbital (LUMO). The LUMO is now localized on the $\mathrm{CF}_{3}$-I bond (Fig. 5), likely a bi-molecular process. The study with a node between the carbon and iodine atoms. Hence, upon a nucleophilic attack this bond will break more easily, thus rendering an explanation for the enhanced reactivity of $\mathbf{1}$ observed in an acidic medium.

In view of the optimization of the reagent, the mechanism of the trifluoromethylation involving benzotriazole and thiophenol was explored computationally. Similarly to reagent $\mathbf{2}$, the assumption is that the $\mathrm{CF}_{3}$ group is transferred to the nucleophile following a reductive elimination (RE) mechanism. If performed in acetonitrile, the first and rate-determining step is the transfer of $\mathrm{CF}_{3}$ to a solvent molecule. The resulting nitrilium intermediate will then react very rapidly with the benzotriazole to form the final product.

The computational study of the reaction performed by Pinto de Magalhães ${ }^{[5]}$ revealed that in gas phase the RE is challenged by an $\mathrm{S}_{\mathrm{N}} 2$ type reaction which leads to the same intermediate product, i.e. nitrilium ions, but at a much lower barrier. Modeling the solvent effect using a polarizable continuum model (PCM) would reduce the gap between the two barriers from 15 to $7.5 \mathrm{kcal} / \mathrm{mol}$ still favoring the $\mathrm{S}_{\mathrm{N}} 2$ mechanism. ${ }^{[6]}$ Sala later showed that the solution to this issue is beyond the limits of current static quantum chemical models. ${ }^{[15]}$ AIMD simulations showed that the RE mechanism is indeed slightly favored,[16] thus confirming the experimental hypothesis. ${ }^{[2]}$ The explicit account of the thermodynamic corrections in the simulation, i.e. the improved description of the contributions of entropy, in particular the solvent

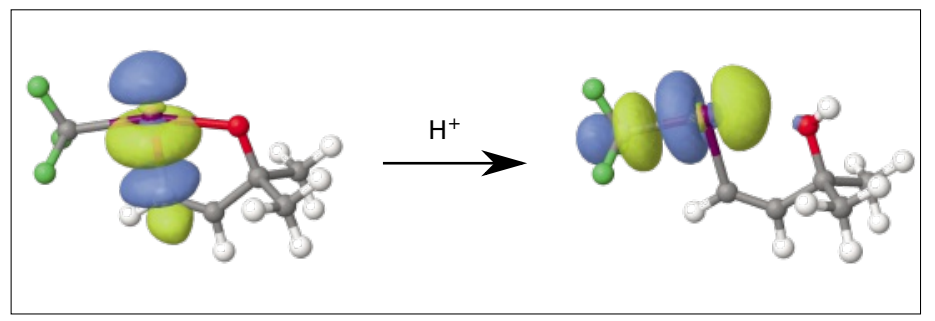

Fig. 5. The change in character of the lowest acceptor level (LUMO) upon protonation. The compound shown here is a model for 1 (vinyl instead of phenyl group bound to iodine). 
entropy contributions (electrostriction, dielectric saturation), are the main reasons favoring the RE over the $\mathrm{S}_{\mathrm{N}} 2$ mechanism.

This example shows that reaction mechanisms explored in gas phase may lead in the wrong direction. However, an AIMD study like the one presented here (Fig. 6), takes months to complete: the mechanistic modelling effort may become very demanding.

The AIMD simulations also revealed that in this reaction a radical mechanism may be involved. Experimentally, radical mechanisms were discovered in the trifluoromethylation of thiophenol using reagent $\mathbf{1}$ to form an $\mathrm{S}-\mathrm{CF}_{3}$ bond. ${ }^{[2,14]}$ As part of his MSc thesis, ${ }^{[7]} \mathrm{S}$. Jungen explored this reaction computationally and presented an alternative radical mechanism (Scheme 1).

In this mechanism, a proton will be transferred from thiophenol 7 to the reagent $\mathbf{1}$ leading to the intermediate $\mathbf{8}$, which can either undergo an RE, or, equally likely, homolytic cleavage of the now rather weak I$\mathrm{CF}_{3}$ bond. The computations show that this process is favored over heterolytic cleavage, and that the case of homolytic cleavage the unpaired electron is localized on the $\mathrm{CF}_{3}$ group 9. Next, homolytic cleavage of the $\mathrm{I}-\mathrm{S}$ bond and recombination of the resulting radicals to form the final product is postulated. This mechanism avoids high barrier transition states, and will be further investigated using AIMD. Even though not yet quantitatively understood, this example again illustrates the mechanistic versatility of iodane reagents.

In the context of this research, a large number of computations have been performed. To address the data processing issue faced when exploring the properties of large arrays of chemically modified reagents, we developed an infrastructure which allows to transfer the data (results) from quantum chemical calculations into a document data base (TURBOMOLE-XML-eXist;[17]). Once archived, the XML documents (one document per calculation), can be queried using XPath/XQuery (Fig. 7). Having the data available in a structured format greatly facilitates their analysis and exchange with other research groups. ${ }^{[18]}$ This infrastructure will be used to find other structure property relationships in iodanes (equilibrium and transition state geometries, ground- and excited state). Advanced data processing is one of the prerequisites to find "something new that works", i.e. to guide experiment in the search for novel materials. One illustration of quantum chemical computation guiding the search for novel materials is the Harvard Clean Energy Project which created a database covering more than three million of prospective organic light harvesting compounds. ${ }^{[19]}$

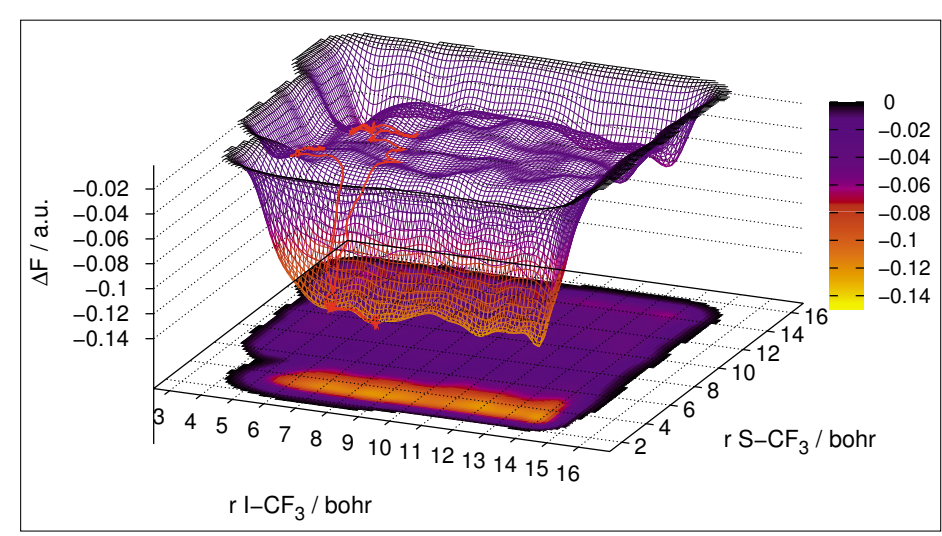

Fig. 6. Free energy surface obtained for the reaction of $\mathbf{1}$ with thiophenol in $\mathrm{MeCN}$ solution as a function of two reaction coordinates (collective variables). Two minimal energy pathways (red lines) represent two possible reaction paths, one of which is an RE.

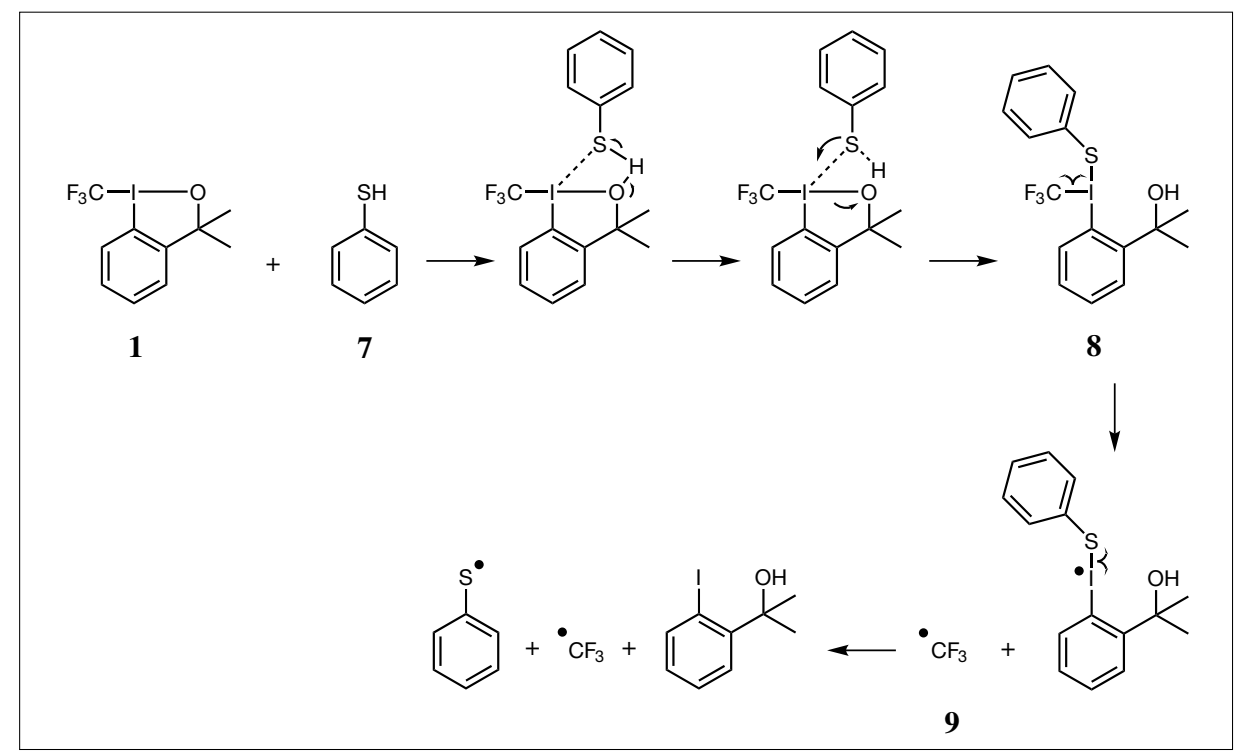

Scheme 1. Proposed radical mechanism for the conversion of thiophenol 7 with reagent 1.

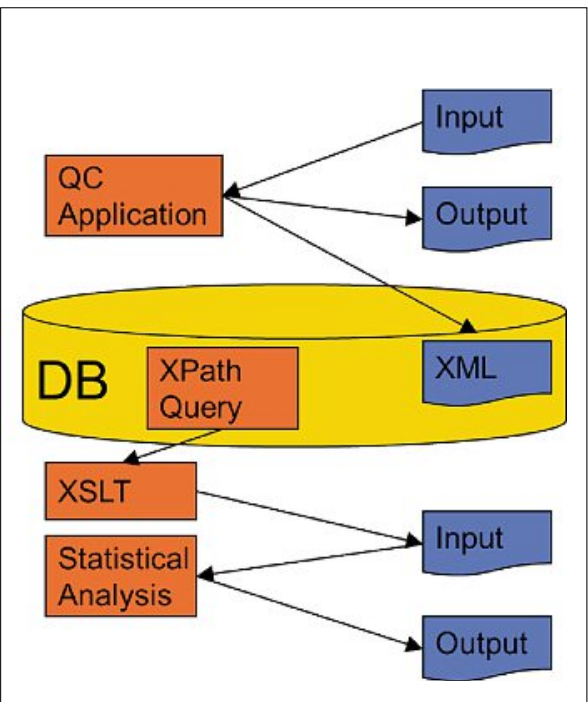

Fig. 7. Processing the data (results) of quantum chemical calculations: the application program (TURBOMOLE), next to the general output file, also generates a data file in XML format, which will be imported into a document data base such as eXist. Once available, the data can be either inspected manually using XPath / XQuery language, or a selection of data can be transformed using extensible stylesheet language (XSLT) into the input for an analysis package.

\section{Conclusions}

The reactivity of iodanes is based on a rich electronic structure reminiscent of the one of transition metals. Given the combination of an interesting chemistry and complex structure and bonding features makes hypervalent iodine compounds ideal topics for the collaboration of modeling and experiment.

Many of the electronic features observed can be expressed in terms of simple orbital considerations. One example presented in this article is the effect of the protonation of $\mathbf{1}$ on its acceptor frontier orbitals and the resulting change in reactivity. There are a number of observations that can be expressed in terms of quantitative structure-property relationships (QSPR). An example is the out-of-plane distortion of the iodine reactive center in reagents with bridged ligands and its impact on the barrier of the functionalization, a QSPR that can be traced back to a pseudo JahnTeller effect.

Whereas these considerations can be communicated on the back of an envelope, some of the predictions which call for quantitative accuracy may be very demanding 
and time consuming. The example shown is the investigation of the reaction mechanism of reagent $\mathbf{1}$ with acetonitrile where the gas phase predictions lead in the wrong direction $\left(\mathrm{S}_{\mathrm{N}} 2\right.$ mechanism), and only the explicit treatment of the solvent by means of an AIMD simulation gives the correct result.

With the understanding of the reactivity of the iodane reagents achieved in this research, the rational design of descendants of these reagents and the exploration of new chemistries is greatly facilitated. "Knowing how it works" will help take the next step, namely to find answers to "what works". On the other hand, progress in chemical research does not depend on complete knowledge of all details: the crystal structure of reagent $\mathbf{6}$, discovered in the early eighties ${ }^{[10]}$ and heavily used ever since, was determined only very recently. ${ }^{[20]}$

\section{Acknowledgements}

This research was supported by the Swiss Science Foundation and has greatly benefitted from the close collaboration with Marcella Iannuzzi and Jürg Hutter of the University of Zurich.

Received: June 27, 2014

[1] a) P. Eisenberger, S. Gischig, A. Togni, Chem. Eur. J. 2006, 12, 2579; b) N. Santschi, A. Togni, Chimia, 2014, 68, 1.

[2] a) J. Carpentier, N. Früh, A. Togni, 2014, Chem. Rev., in revision; b) N. Santschi, A. Togni, Chimia 2014, 68, 419.

[3] V. V. Zhdankin, P. J. Stang, Chem. Rev. 2008, $108,5299$.

[4] R. Helbling, MSc. Thesis (University of Zürich), 2009.

[5] H. Pinto de Magalhães, MSc. Thesis (ETH Zürich), 2011.

[6] O. Sala, MSc. Thesis (ETH Zürich), 2012.

[7] S. Jungen, MSc. Thesis (ETH Zürich), 2014

[8] H. Pinto de Magalhães, H. P. Lüthi, A. Togni, Org. Lett. 2012, 14, 3830.

[9] J. Malmgren, S. Santoro, N. Jalalian, F. Himo, B. Olofsson, Chem. Eur. J. 2013, 19, 10334.
[10] a) D. B. Dess, J. C. Martin, J. Org. Chem. 1983, 48,4155 ; b) D. B. Dess, J. C. Martin, JACS, $1991,113,7277$.

[11] B. Wang, J. W. Graskemper, L. Qin, S. G. DiMagno, Angew. Chem., Int. Ed. 2010, 49, 4079.

[12] H. Pinto de Magalhães, H. P. Lüthi, A. Togni, J. Org. Chem., DOI: 10.1021/jo501714f.

[13] V. R. Grushin, Chem. Soc. Rev. 2000, 29, 315.

[14] N. Santschi, PhD Thesis (ETH Zürich), 2014.

[15] O. Sala, H. P. Lüthi, A. Togni, J. Comp. Chem., in press.

[16] O. Sala, H. P. Lüthi, A. Togni, M. Iannuzzi, J. Hutter, to be published.

[17] A. Glöss, M. P. Brändle, W. Klopper, H.P. Lüthi, Mol. Phys. 2012, 110, 2523

[18] M. Brändle, P. Bühlmann, C. C. M. Samson, A. Elsener, H. P. Lüthi, Chimia 2007, 61, 165.

[19] J. Hachmann, R. Olivares-Amaya, A. Jinich, A. L. Appleton, M. A. Blood-Forsythe, L. R. Seress, C. Román-Salgado, K. Trepte, S. Atahan-Evrenk, S. Er, S. Shrestha, R. Mondal, A. Sokolov, Z. Bao, A. Aspuru-Guzik, Energy Environ. Sci. 2014, 7, 698

[20] A. Schröckeneder, D. Stichnoth, P. Mayer, D. Travner, Beilstein J. Org. Chem. 2013, 8, 1523. 